\title{
What will research tell us about the future in IBD?
}

\author{
STEPHEN M COLLINS, MB,BS, FRCP(UK), FRCPC, KENNETH CROITORU, MDCM, FRCPC
}

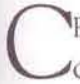
ROHN'S DISEASE AND ULCERATIVE colitis are referred to collectively as 'inflammatory bowel disease' (IBD) and are characterized by inflammation in the gut. Basic research in IBD is aimed at understanding the pathogenesis and pathophysiology of these conditions. Pathogenesis refers to the primary cause of the disease and pathophysiology refers to understanding the disease process once it is established. It follows that success in understanding the pathogenesis of the disease may ultimately lead to a cure or at least prevention and an understanding of the pathophysiology will lead to the development of more selective and effective treatments for established disease. Pathogenesis of the disease leads to the pathophysiology which, in turn, produces symptoms and disability. Research at a clinical level is aimed at improving management of the disability incurred by IBD; this involves evaluation of new therapies, the refinement of surgical techniques and obtaining a better understanding of how the disease process in general impacts on the quality of life of patients and their families.
In terms of understanding better the pathogenesis of IBD, current research addresses three main issues. First, is there a defect in the immune system of the intestine, rendering individuals susceptible to IBD? Secondly, is part of this susceptibility inherited? Thirdly, are there any identifiable factors in the environment that play an important role in the initiation of the disease process. These issues are not mutually exclusive and it is probable that in most IBD patients, two or more of these factors are important.

\section{IS THERE A DEFECT IN THE IMMUNE SYSTEM?}

There is plenty of experimental evidence showing that immune function is altered in the intestine of patients with IBD. There are changes in the distribution of a variety of important immune cells, particularly lymphocytes. Lymphocytes are of two major types, T lymphocytes and B lymphocytes. B lymphocytes produce antibodies that help fight disease and these antibodies are present in tissues and fluids throughout the body. They are

Intestinal Diseases Research Unit, Division of Gastroenterology, McMaster University, Hamilton, Ontario

Correspondence and reprints: Dr Stephen M Collins, McMaster University Medical Centre, Room 3N5C, Hamilton, Ontario L8N 325. Telephone (4I6) 525-9140

directed against chemicals 'foreign' to the body that may occur as part of viruses, bacteria, drugs or nutrients. There are several categories of antibody and each fulfils a specific need. When $\operatorname{IgG}$ is produced, it not only blocks the foreign protein or chemical against which it is directed, but it alerts the body's defences to produce an inflammatory reaction.

Because the gut sees lots of foreign substances on a daily basis, it is important in some way to 'soften' the antibody response to the environment and there is a specific type of antibody that occurs on delicate surfaces, such as that lining the gut. This type of antibody is called $\operatorname{IgA}$ and its production is not associated with much inflammation. If, for some reason, individuals produce relatively more $\operatorname{IgG}$ than $\operatorname{IgA}$, then clearly they are at risk of 'over-reacting' to foreign chemicals and to initiating an inflammatory process in the gut. An imbalance between these antibody types has been seen in IBD patients but, like all other abnormalities, the million dollar question is whether this occurs as a primary event or whether it occurs secondary to the disease process itself. It is hoped that further research will clarify this.

T lymphocytes play a very important role not only in defending against 
foreign material, but also in generally controlling the body's immune response. $\mathrm{T}$ lymphocytes come in two major subsets, the $T$ helper cell which serves to control the immune response in and the $T$ cytotoxic cell which reacts directly to foreign material. The focus of a great deal of very exciting research in the past few decades has been toward the identification of the molecule on $T$ cells responsible for recognizing foreign material. The equivalent of the antibody on the $\mathrm{T}$ cell, the $\mathrm{T}$ cell receptor, has now been identified and its genes isolated.

The examination of $\mathrm{T}$ cells in IBD has shown that although there are increases in their numbers, there does not seem to be a major change in the distribution of the different $T$ cell subsets. Furthermore, work at trying to identify an abnormal $\mathrm{T}$ cell, reactive against one's own intestinal cells, has not been successful. Much of this work, however, preceded the identification of the $\mathrm{T}$ cell receptor,

The future of research on the role $T$ cells play in the pathophysiology and pathogenesis of IBD will involve the examination of the ever-increasing numbers of molecules produced by $\mathrm{T}$ cells and how they effect the immune response, and the direct examination of the $\mathrm{T}$ cell receptor molecule and its genes for the possibility that this may provide the means of identifying the initiating event in IBD. The new and exciting techniques of cell and molecular biology will provide the tools for examining the role both $\mathrm{T}$ and $\mathrm{B}$ cells play in IBD.

\section{IS THERE A RECOGNIZABLE HEREDITARY DEFECT?}

It has long been recognized that there is an hereditary component to IBD. Between 10 and $20 \%$ of IBD patients have a positive family history of the disease. In addition, there is a higher than expected incidence of IBD in identical twins. IBD is prevalent among Jewish populations throughout the world and is particularly prevalent among Ashkenazi Jews. These observations strongly suggest that there is a genetic component to IBD. The inherited component in itself is unlikely to cause disease but it may take the form of a predisposition or weakness to the development of the disease which requires the presence of other factors. Thus, one may inherit the susceptibility without necessarily getting the disease.

In order to understand both the inheritance pattern and the nature of the susceptibility it is necessary to obtain as much information as possible, not only from patients with IBD but also from their relatives and cohabitants. Future studies will involve both clinical observation and special testing of blood and tissues from such individuals. A study has already begun on an evaluation of abnormal permeability of the intestine in such groups. A study from California has suggested that relatives of patients with IBD may have a 'leakiness' of the bowel wall (permeability) that may predispose them to the entry of large proteins which normally would be excluded from a tightly knit gut wall (1). This remains a provocative but controversial finding and further research must clarify whether this is indeed the case. Along the same lines, studies on immune markers and other aspects of intestinal function need to be performed if we are to understand the inheritance pattern and the nature of the inherited predisposition. To achieve this objective, it is necessary to evaluate large numbers of patients and their relatives. This should be done at least at a provincial level, and ideally at a national level with generation of a large database to which all major centres dealing with IBD cases contribute.

\section{CAN WE IDENTIFY INITIATING FACTORS IN THE ENVIRONMENT?}

It is likely that certain individuals are predisposed to the development of IBD. Occurrence of the disease depends on a variety of factors that may include both inheritance patterns and factors in the environment. Studies to date have been unsuccessful in identifying an infective agent (virus, bacteria, etc) that causes IBD. In the past, much attention focused on the bacteria responsible for tuberculosis. There are certain features of intestinal tuberculosis that are similar to Crohn's disease. How ever, there has never been any convincing report of transmission of IBD between individuals and the responses of IBD patients to treatment with antimicrobial or antituberculous drugs have not been consistent. These findings do not dismiss a role for infective agents, tuberculosis-related organisms or others from a role in the initiation of IBD.

At this time, there is a viable hypothesis that states that in certain predisposed individuals, infection with a hitherto unidentified organism produces an inflammatory and immunologic response in the gut; whereas in normal subjects this would result in clearance of the organism and resolution of the inflammatory process, in predisposed individuals the immunologic response to the organism becomes directed towards an immunologic response to various normal components of the gut wall. That is, following the initial infection, the body's immune system views these components of the gut as being 'foreign' and perpetuates an ongoing attack against them. This idea is based on the notion that certain proteins presented by organisms such as bacteria are chemically similar to certain proteins in the gut wall. When the organism invades the gut, the immune system directs an attack against the organism's proteins and, in so doing, starts to attack normal tissues in the body. Since this does not happen in all individuals, the implication is that predisposed subjects may either have unusual protein constituents in their gut wall or, more likely, have a problem in the ability of their immune system to finely distinguish foreign proteins from normal constituents of the body.

Factors other than infection have been considered to play a role in the development of IBD and these include various dietary factors, drugs and social habits such as smoking. Previously, much attention was paid to the fact that IBD patients often demonstrated antibodies towards milk protein and it was felt that there may be some hypersensitivity to milk. However, it is currently believed that this occurs as a secondary phenomenon, the leakiness 
of the inflamed bowel permitting entry of foreign (dietary) proteins including milk into the body where an appropriate immune response is mounted. There is no good evidence that milk is an initiating or aggravating factor in most cases of IBD. The role of cigarette smoking and various drugs such as the contraceptive pill and nonsteroidal anti-inflammatory drugs is presently being examined. Further research is required to confirm clinical suspicion of an association between these factors and the development of the disease and, again, studies on large populations of patients are required.

Epidemiological surveys provide very important information about the possible link between environmental factors and the development and exacerbation of IBD. Once a factor, whether it is a dietary protein, a habit such as smoking or a drug, has been linked to the disease, it is then necessary to examine how the factor might initiate or exacerbate an inflammatory process in the intestine and this usually requires animal studies. Thus, future research will tell us a lot more about the link between what we ingest, inhale and do, and the development and modulation of IBD. As these relationships are catalogued in clinical (epidemiological) studies, we can begin to focus on certain common features of these associations, and then to develop hypotheses regarding how they relate to the inflammatory process in the bowel, and ultimately to test them in experimental situations. Positive results in this area will ultimately lead to a better understanding of the cause of IBD; in the meantime, information obtained from these studies will help guide physicians in their counselling of IBD patients with respect to lifestyle.

Another aspect of lifestyle that is frequently raised in discussion of IBD is the role of stress. While the scientific data confidently linking stress with the development and exacerbation of IBD are inconclusive at this time, it is the experience of most patients and many physicians that such a relationship indeed exists. While it is generally appreciated that stress is harmful and aggravates any existing disease, and this includes heart disease, hypertension, asthma, etc, there is a notion that the gut may be particularly prone to the consequences of stress. This is based on the recognition that bowel symptoms occur frequently in healthy subjects subjected to stress, that a very common clinical disorder called irritable bowel syndrome (spastic colon) is thought to be, at least in part, stress-induced, and that the gut and the brain have a special and intimate relationship.

The brain and the gut are connected by a network of nerves and the gut itself has its own nervous system, the myenteric plexus ('mini brain'). In fact, there are as many nerve cells in the gut as there are in the spine. We are only just beginning to understand the organization and function of the mini brain, but it is becoming clear that it not only transduces signals from the brain to the gut, but also relays information about the environment from the gut to the brain. Thus, there is not only a mechanism whereby signals from the brain can alter gut function, but also a mechanism whereby changes in gut function can be transmitted to the brain. The gut can be viewed as much as a sensory organ as the skin, detecting changes in the environment and transmitting them to the brain.

An understanding of the role of the gut as a sensory organ is in its infancy but research in the future should generate considerable new information about the sensory role of the gut, and how this role may be altered in disease. Equally important is the renewal of research interest in how the brain modulates immune function. Results of research performed in the past five to 10 years provide a clear indication that the mini brain has the ability to alter the function of immune cells in the gut. Again, this is an area that is in its infancy but one which may have a dramatic effect on understanding the relationship between behaviour and disease activity in the gut. Clearly, if the immune system in the gut receives information from the brain, then it is not difficult to envisage how stress and behavioral alteration may influence inflammatory processes in the gut. It is, however, another thing to suggest that behavi- oral abnormalities may actually cause the inflammatory disease process. Although evidence is emerging from other tissues (such as skin) that nerve stimulation itself may lead to a sequence of events resulting in inflammation (neurogenic inflammation), there is no evidence that neural stimulation, either at the level of the brain or the mini brain, can lead to an inflammatory process similar to that seen in IBD.

\section{PATHOPHYSIOLOGY OF IBD}

We have discussed how individuals might be predisposed to IBD, and how an abnormal intestinal immune system might overreact in response to factors initiated from the environment, to produce intestinal inflammation and, thus, IBD. Once this process is initiated, and the bowel becomes inflamed, symptoms are generated and disability incurred. While the search continues for the cause, and thus the cure for the disease, it is important to understand better the inflammatory process itself within the gut. Research in this area is likely to yield new information that will help us better control the inflammatory process and, in so doing, provide more effective therapy for symptoms and minimize disability.

It is likely that alterations in immune function play a primary role in the pathogenesis of IBD. However, alteration in immune function can be considered in another context, namely the perpetuation of the disease. Under normal circumstances, when inflammation occurs in response to an external event such as an infection, it is usually limited in that the inflammatory response subsides once the initiating event is over. In IBD, there appears to be a problem with the control of the inflammatory process in that what starts as acute inflammation goes on to become chronic and relapsing. We do not understand why the inflammatory process appears to lack control and self-containment. One possibility is that the initiating factor(s) continues to be present; this, on the basis of epidemiological study and patient observation, appears to be unlikely. The most likely explanation is a defect in the regulation of the inflammatory 
response. Research on the nature of the inflammatory process in the intestine continues to yield new information and attention is being paid to understanding the transition between acute and chronic inflammation using a variety of animal models. Future research therefore is expected to reveal something about the signals that normally herald the reduction in the inflammatory process. This information could also be used to try to minimize relapses in patients with established disease.

Once the immune system is activated, there is an orchestration of this large number of different inflammatory cell types. The orchestration of these cells into an inflammatory response requires a large repertoire of chemicals known as inflammatory mediators or cytokines. These are chemicals that transmit information between immune or inflammatory cells, and that results in the recruitment of a large number of other inflammatory cells as well as changes in the surrounding tissues. Examples of these chemicals include prostaglandins, leukotrienes, interleukins. The past decade has seen an explosion of knowledge in this area with the identification of large numbers of new inflammatory mediators or interleukins.

As research in this area progresses, new information as to how these chemicals alter normal cell processes will be forthcoming. More importantly, as each mediator is identified, it is hoped that chemicals will be developed that will block or inhibit the actions of these inflammatory mediators. These chemical 'blockers' will form the basis of a whole new generation of drugs that can control the inflammatory process and achieve better symptomatic control in IBD. Since inflammatory mediators change a variety of functions, including the ability of the bowel to absorb water, salts and nutrient and the ability of muscle to propel nutrients and fecal matter through the gut, it is likely that this new generation of 'blocking' chemicals will benefit a wide variety of IBD symptoms. Also, there is current interest in how the gut is involved in the regulation of appetite, both under normal conditions and following inflammation. Since a loss of appetite is very common in IBD, particularly in children where it may result in malnutrition, it is important that the mechanism underlying this process be understood. Again, it is probable that the suppression of feeding may well be due to an inflammatory mediator and thus a better understanding of these events, together with the development of 'blocking' chemicals, may help improve this important aspect of IBD.

\section{CONCLUSIONS}

The present research thrust in IBD is broad based and no longer seeks a single cause or explanation for the disease process. Rather, it embraces a much broader hypothesis regarding the cause of IBD. Our present thinking is that IBD results from the convergence of several factors in susceptible individuals and the combination of these factors may vary between individuals with IBD. Thus, the IBD patient population may be quite heterogeneous or comprise a number of subpopulations. The approach to IBD research these days recognizes the need to document and investigate large numbers of patients and it is felt that this should be done collectively, at a provincial or, ideally, a national level. It is important that researchers share information obtained from their respective IBD populations in order to achieve this goal.

Research, particularly in the area of mucosal immunology, is likely to yield important new information regarding the nature of abnormalities that not only predispose to the development of IBD, but also perpetuate the inflammatory process. An appreciation of the interaction between the brain and the immune system, through the mini brain in the gut, may help to clarify the relationship between behaviour and disease activity. Finally, a dramatic increase in understanding of the chemicals that mediate the inflammatory process is expected. In the short term. this will provide better tools to treat symptoms and therefore minimize disability in patients with established IBD. Regardless of the research topic involved, each of these endeavours requires the continued close cooperation between patients and their physicians and the Canadian Foundation for Ileitis and Colitis (CFIC). Research in IBD is neither the exclusive domain of clinicians nor basic scientists but rather an opportunity for researchers from these backgrounds to share their talents and observations in order to provide a better understanding of IBD.

\section{REFERENCE}

1. Katz KD, Hollander D, Vadheim CM, et al. Intestinal permeability in patients with Crohn's disease and their healthy relatives. Gastroenterology 1989;97:927-31. 


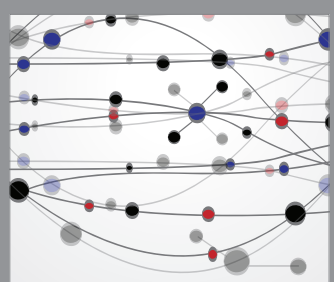

The Scientific World Journal
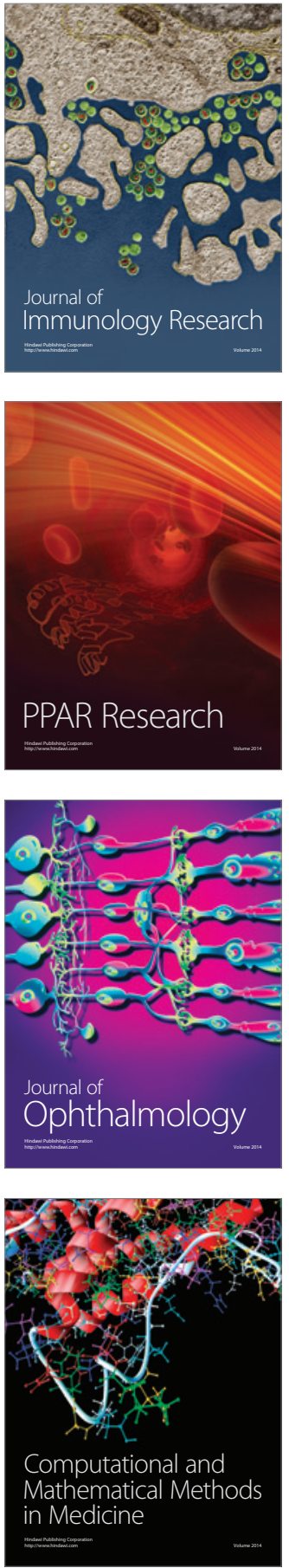

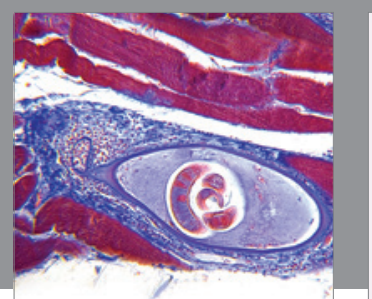

Gastroenterology Research and Practice

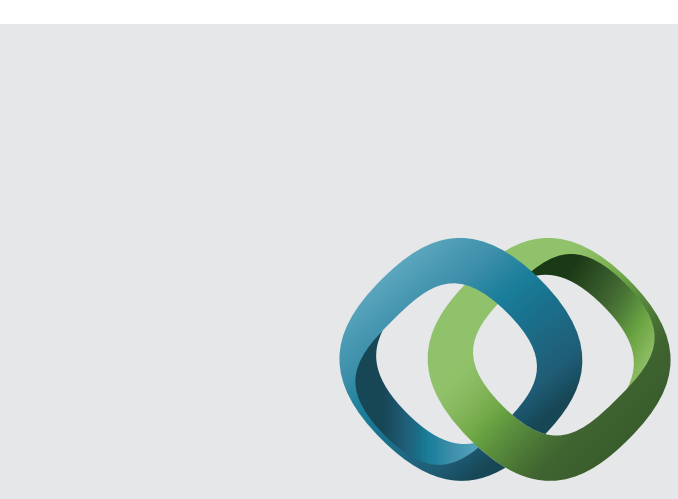

\section{Hindawi}

Submit your manuscripts at

http://www.hindawi.com
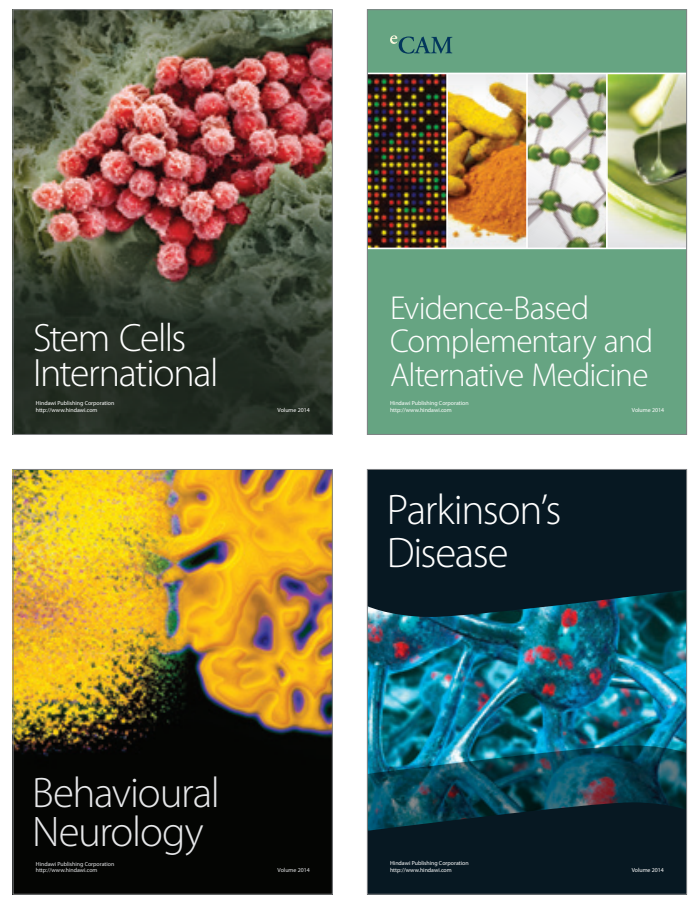
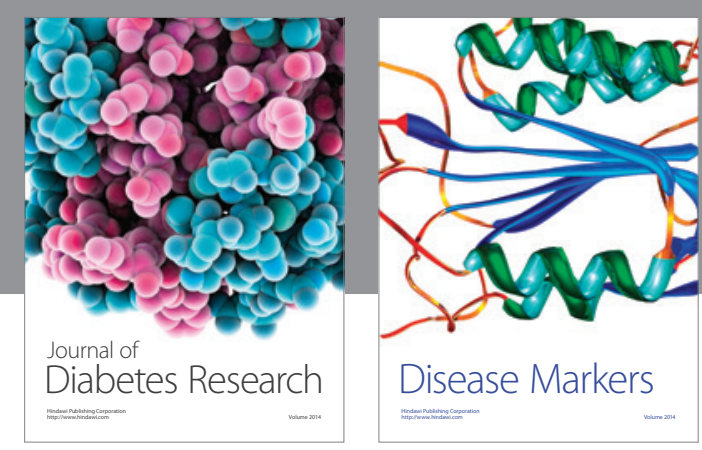

Disease Markers
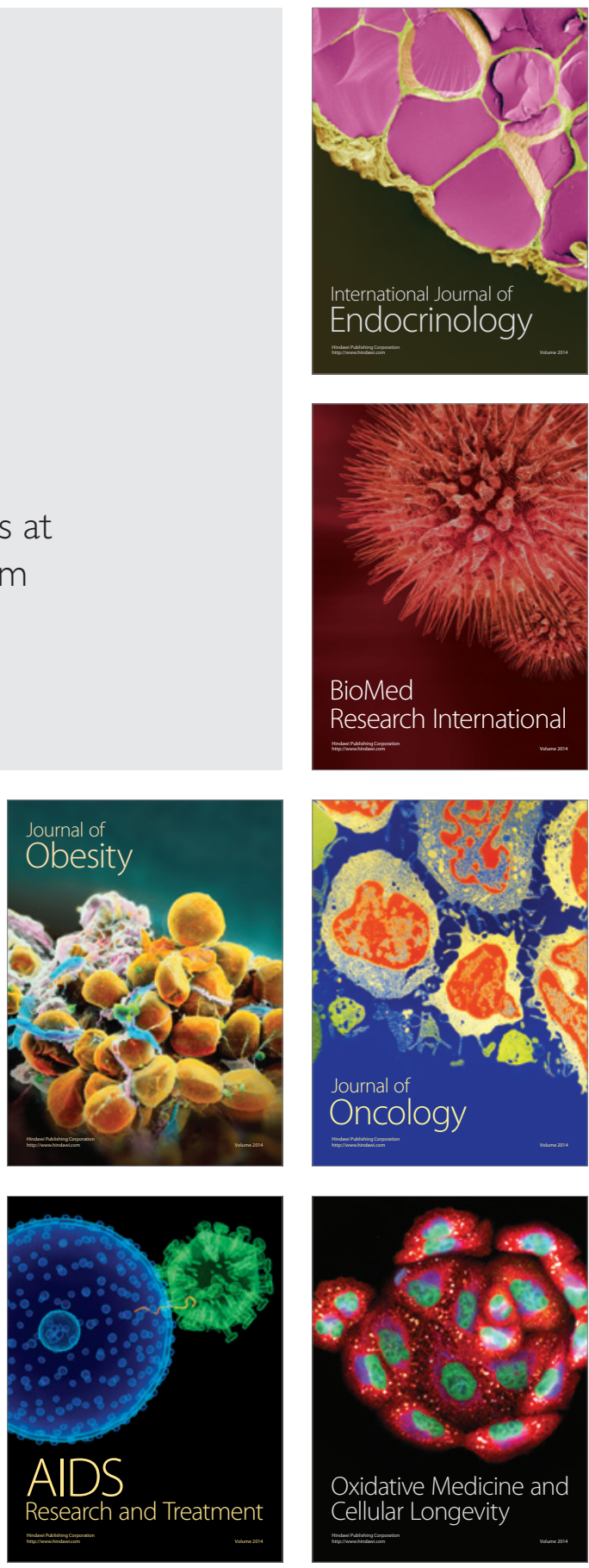